Forum $2022 \cdot 37: 7-8$

https://doi.org/10.1007/s12312-021-01033-9

(c) The Author(s), under exclusive licence to Springer Medizin Verlag $\mathrm{GmbH}$, ein Teil von Springer Nature 20212021

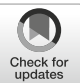

\section{COVID-19: geimpft, genesen oder erkrankt}

\author{
Thomas Seufferlein \\ Klinik für Innere Medizin I, Universitätsklinikum Ulm, Ulm, Deutschland
}
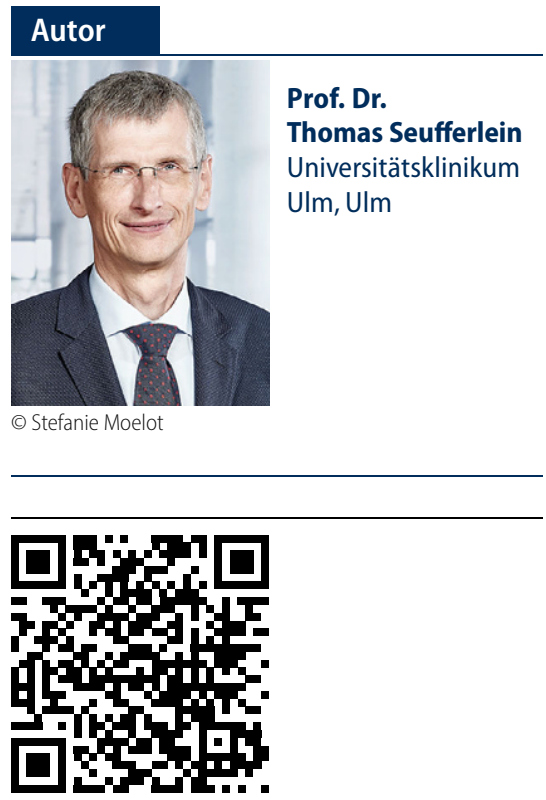

QR-Code scannen \&Beitrag online lesen
Allen mahnenden Rufen aus der Wissenschaft zum Trotz - die vierte Welle der COVID-19-Pandemie erzeugt Ende 2021 Inzidenzraten, die alles bisher Dagewesene deutlich übersteigen. Durch die deutlich infektiösere Omikron-Variante verschärft sich die Lage zusätzlich. Und wieder, wie bereits im Jahr zuvor, bringt die hohe Zahl an COVID-Infektionen das medizinische und pflegerische Personal in Deutschland an seine Belastungsgrenze. Die drei Partnerorganisationen der Corona-Taskforce, das Deutsche Krebsforschungszentrum, die Deutsche Krebshilfe und die Deutsche Krebsgesellschaft, nahmen diese Entwicklung zum Anlass, sich Ende November 2021 für Kontaktbeschränkungen und eine allgemeine Impfpflicht auszusprechen.

Dabei waren die Warnungen der Expert*innen vor einer vierten COVID-19Welle am Ende des Sommers 2021 klar. Die politischen Konsequenzen folgten leider dem Leitsatz "Es wird schon nicht so schlimm kommen“. Den Unentschlossenen unter den Ungeimpften signalisierte man durch das Beenden der epidemischen Lage von nationaler Tragweite und die Ankündigung des Freedom Day, dass die Pandemie überwunden sei. Impfzentren wurden geschlossen, eine rasche Durchführung von groß angelegten Impf- und Booster-Kampagnen ist deshalb jetzt schwierig. 2- oder 3G-Regelungen in Restaurants oder Bars? Oft genug lax gehandhabt, sowohl von Betreibern als auch von Gästen. 3G-Regelungen in der Arbeitswelt wurden erst am 24.11. für alle Arbeitgeber verpflichtend. Und mit der Einführung der Hospitalisierungsinzidenz hat man zudem die Verschärfung von Coronamaßnahmen von einem Indikator abhängig gemacht, der die Situation der Krankenhäuser erst mit deutlicher Verzögerung und zudem un- vollständig anzeigt. Andere europäische Länder, wie etwa Spanien oder Italien, haben - nicht zuletzt aufgrund ihrer deutlich höheren Impfquote - die vierte COVID-19Welle sehr viel besser im Griff als Deutschland.

Als Ärzt*innen und Wissenschaftler*innen setzen wir auf Daten, Zahlen, Fakten und Forschungsergebnisse, um unseren Patient*innen zu helfen. Mittlerweile stehen uns wirksame Impfstoffe zur Eindämmung der Pandemie zur Verfügung, und die ersten Medikamente gegen COVID-19 sind zugelassen. Die Pandemie sollte also an Schrecken verloren haben. Trotzdem fordert sie noch immer unnötig Menschenleben. Sie zwingt uns dazu, Behandlungen zu priorisieren. Sie erhöht die Zahl Krebskranker, die aufgrund zu später Behandlung eine schlechtere Prognose haben. Sie verschleißt die Kräfte des ärztlichen und pflegerischen Personals dauerhaft und verursacht wirtschaftliche und soziale Schäden. Ich würde mir wünschen, dass wissenschaftliche Warnungen mehr Gehör in der Politik finden. Viele der genannten Vorteile wurden in den vergangenen Monaten durch ein zu unentschlossenes Krisenmanagement und teilweise schlichtes Nichtstun verspielt. Für mich steht aber auch fest: Der Freiheitswunsch des Einzelnen hört da auf, wo er das Wohl der Allgemeinheit gefährdet. In einer Bevölkerung, die von sich aus Maske trägt, sich in Scharen impfen lässt, und das eigene Freiheitsbedürfnis kurzfristig hintanstellt, um als Ganzes bald wieder mehr Freiheit zu erlangen, wäre die Forderung nach einer allgemeinen Impfpflicht nicht nötig.

Ihr Thomas Seufferlein

Präsident der Deutschen Krebsgesellschaft 
Korrespondenzadresse

Prof. Dr. Thomas Seufferlein

Klinik für Innere Medizin I, Universitätsklinikum Ulm

Albert-Einstein-Allee 23, 89081 Ulm,

Deutschland

thomas.seufferlein@uniklinik-ulm.de

\section{Einhaltung ethischer Richtlinien}

Interessenkonflikt. T. Seufferlein gibt an, dass kein Interessenkonflikt besteht.

Für diesen Beitrag wurden von den Autoren keine Studien an Menschen oder Tieren durchgeführt. Für die aufgeführten Studien gelten die jeweils dort angegebenen ethischen Richtlinien.

\section{Curt Meyer-Gedächtnispreis 2022}

Die Berliner Krebsgesellschaft e.V. vergibt den Curt Meyer-Gedächtnispreis verbunden mit einem Preisgeld in Höhe von 10.000 EUR zur Förderung des wissenschaftlichen Nachwuchses im Land Berlin.

Prämiert wird eine wissenschaftliche Persönlichkeit, die sich mit ihrer Forschungstätigkeit auf dem Gebiet der Krebsmedizin aktuell hervorgetan hat. Durch das Herausstellen von Vorbildern treibt die Berliner Krebsgesellschaft die Spitzenwissenschaft im Bereich der Krebsmedizin voran.

\section{Voraussetzungen}

Der/die Bewerber:in darf nicht älter als 40 Jahre und muss im Land Berlin beruflich tätig sein. Bei Inanspruchnahme von Mutterschutz- und Elternzeiten kann eine Verschiebung der Altersgrenze um zwei Jahre pro Kind gewährt werden.

In einem Begutachtungsverfahren wird der/die Preisträger:in auf der Grundlage von einer oder mehreren hochrangig publizierten Arbeiten bestimmt. Hierfür können Publikationen eingereicht werden, die sich mit Forschungsfragen auf dem Gebiet der klinischen, experimentellen oder translationalen Krebsmedizin sowie der Versorgungsforschung befassen, wenn diese an einer Institution des Landes Berlin erarbeitet wurden. Die Artikel müssen aktuell sein. Wurde das Manuskript einem Verlag zur Veröffentlichung vorgelegt, muss das Begutachtungsverfahren abgeschlossen und das Manuskript zur Veröffentlichung angenommen worden sein. Eine Nominierung ist nicht erforderlich. Die Vergabe des Preises erfolgt an den/die Erstautor:in. Die Koautor:innen müssen schriftlich bestätigen, dass sie mit der Bewerbung des/der Erstautor:in einverstanden sind.
Die Bewerbung ist an Prof. Dr. Petra Feyer, Vorstandsvorsitzende der Berliner Krebsgesellschaft e.V., zu richten. Folgende Unterlagen müssen eingereicht werden:

1. Lebenslauf

2. Wissenschaftlicher Tätigkeitsbericht

3. Publikationsliste

4. Publikation/en, die für die Bewerbung relevant sind

Bewerber:innen senden bitte ihre Unterlagen bis spätestens 31. März 2022

(23.59 Uhr) ausschließlich per E-Mail in einer pdf-Datei (nicht größer als 7MB) an: forschung@berliner-krebsgesellschaft.de

\section{Über den Curt Meyer-Gedächtnispreis} Der Curt Meyer-Gedächtnispreis gehört zu den renommiertesten Auszeichnungen für junge Krebsforscher:innen in Berlin. 1988 zum ersten Mal von der Berliner Krebsgesellschaft e.V. verliehen, soll der mit 10.000 Euro dotierte Forschungspreis die Spitzenforschung in Berlin stärken und Nachwuchswissenschaftler zu herausragenden Leistungen animieren. Der Preis dient dem ehrenden Gedächtnis an den Berliner Senatsrat Dr. med. Curt Meyer (1891-1984), dessen Name eng mit der Krebsfürsorge verbunden ist. Meyer war Gründungsmitglied und langjähriger Geschäftsführer des Landesausschusses Berlin für Krebsbekämpfung e.V., aus dem 1957 die Berliner Krebsgesellschaft hervorgegangen ist.

$\because \because: \because:$ :::::: Berliner Krebsgesellschaft e.V. Durch Wissen zum Leben

Quelle: Berliner Krebsgesellschaft e.V., www.berliner-krebsgesellschaft.de 